\title{
A chemical modification/interference study of yeast pre-mRNA spliceosome assembly and splicing
}

\author{
Brian C. Rymond and Michael Rosbash \\ Biology Department, Brandeis University, Waltham, Massachusetts 02254 USA
}

\begin{abstract}
A chemical modification/interference assay was used to determine the yeast pre-mRNA sequence requirements for in vitro spliceosome assembly and splicing. Modifications of any of the nucleotides within the 5 ' splice site and branch point (TACTAAC box) consensus sequences as well as less conserved intron and exon positions were found to inhibit assembly and/or splicing. The interference pattern of the $5^{\prime}$ splice site and TACTAAC box lesions increased as spliceosome assembly proceeded (complex III $\rightarrow$ complex I $\rightarrow$ complex II) and as splicing proceeded, suggesting that these sequence elements play multiple roles in the assembly of yeast spliceosomes and in the removal of intervening sequences. Furthermore, modification (or mutation) of a TACTAAC-like sequence upstream of the branch point was found to inhibit the rate of spliceosome assembly, implying a possible role for degenerate branch point sequences in modulating the efficiency of spliceosome assembly.
\end{abstract}

[Key Words: RNA processing; splicing; spliceosome; Saccharomyces cerevisiae]

Received December 21, 1987; revised version accepted February 8, 1988.

The removal of introns from nuclear pre-mRNA transcripts is preceded by the association of the pre-mRNA with a variety of proteins and RNAs. The resulting macromolecular complex, the spliceosome, presumably facilitates and specifies the bond cleavage and ligation events that characterize pre-mRNA splicing (for review, see Green 1986; Padgett et al. 1986; Maniatis and Reed 1987). In the first step of the splicing reaction, the premRNA is cleaved at the exon I/intron boundary. Concurrently (or in rapid succession) the $5^{\prime}$ terminal nucleotide of the intron is joined via a $2^{\prime}-5^{\prime}$ bond to an adenosine residue (the branch point) near the $3^{\prime}$ end of the intron. The products of this first step land intermediates of the complete reaction) are exon I and the lariat intermediate (composed of the intron in a lariat configuration joined to exon II). In the second step of the reaction, the lariat intermediate is cleaved at the intron/exon II boundary; concomitant (or subsequent) exon ligation generates the mRNA and excised intron products. Whether components of the spliceosome actually cleave the pre-mRNA and ligate the exons during splicing or act as a scaffold for a pair of pre-mRNA-directed transesterification reactions [similar to those that take place in the maturation of the chloroplast and mitochondrial self-splicing, class II introns (for review, see Cech and Bass 1986) is not known. Both models predict an intimate association of the splicing factors with the target pre-mRNA, an assertion for which there is considerable experimental support (Green 1986; Padgett et al. 1986; Maniatis and Reed 1987).

The interaction of yeast splicing factors with pre-
mRNA molecules has been monitored with a variety of techniques, including velocity sedimentation (Brody and Abelson 1985; Lin et al. 1987); gel electrophoresis (Pikielny and Rosbash 1986; Pikielny et al. 1986; Cheng and Abelson 1987); affinity chromatography (Kretzner et al. 1987; Rymond et al. 1987); and nuclease sensitivity (Rymond and Rosbash 1985). The results obtained from these studies suggest that the assembly of spliceosomes progresses in a stepwise fashion. Based on a number of criteria including characterization of the three electrophoretically resolvable forms of the yeast spliceosome (complexes I, II, and III), we have proposed a three-step model for the assembly pathway: complex III $\rightarrow$ complex I $\rightarrow$ complex II = mature spliceosome (Pikielny et al. 1986).

In this study, we assayed the sensitivity of spliceosome assembly and splicing to inhibition by chemically induced pre-mRNA lesions. Modifications of every conserved nucleotide of the $5^{\prime}$ splice site and branch point sequence (TACTAAC box), as well as specific but less conserved nucleotides in exon I and a TACTAAC-boxlike sequence upstream of the branchpoint, inhibited spliceosome assembly, lariat formation, and/or mRNA production. Modification of the $5^{\prime}$ terminal G of exon II only inhibited ligation. In contrast, lesions at the $3^{\prime}$ splice site (or elsewhere in the intron or exons) did not inhibit spliceosome assembly or lariat formation. Consistent with the three-step model of assembly, the modified nucleotides had more severe effects on the later stages of spliceosome assembly than on the earlier ones. Taken together, these results suggest that individual nu- 
cleotides within the conserved consensus sequences may play different and perhaps multiple roles during spliceosome maturation.

\section{Results}

\section{Preparation of modified pre-mRNA}

Yeast (Saccharomyces cerevisiae) pre-mRNA substrates were synthesized by in vitro transcription of the SP6/ RP51A fusion gene pSPrp51A $\Delta 2$. This RNA is a small and efficient substrate for in vitro spliceosome assembly and splicing (Rymond and Rosbash 1985; Rymond et al. 1987).

An outline of the modification/interference assay, illustrated in Figure 1, is analogous to methylation interference studies performed with modified DNA and DNA-binding proteins (Siebenlist and Gilbert 1980). The $3^{\prime}$ ends of the in vitro synthesized pre-mRNA were labeled with $\left.{ }^{32} \mathrm{P}\right] \mathrm{pCp}$ and RNA ligase. Chemical lesions were then introduced with diethyl pyrocarbonate (to modify purines) and hydrazine (to modify pyrimidines) (Peattie 1979). Conditions were established such that, on average, less than one modification was introduced per molecule (data not shown). The modifications (N-6,7 carbethoxylation of A, N-7 carbethoxylation of $G$, and base removal of $\mathrm{C}$ and $\mathrm{U}$ ) appeared randomly distributed along the RNA chain (although in some experiments the uridine and cytosine lesions were not evenly balanced; see Materials and methods). To assay spliceosome formation, we incubated modified, radioactive RNA in a

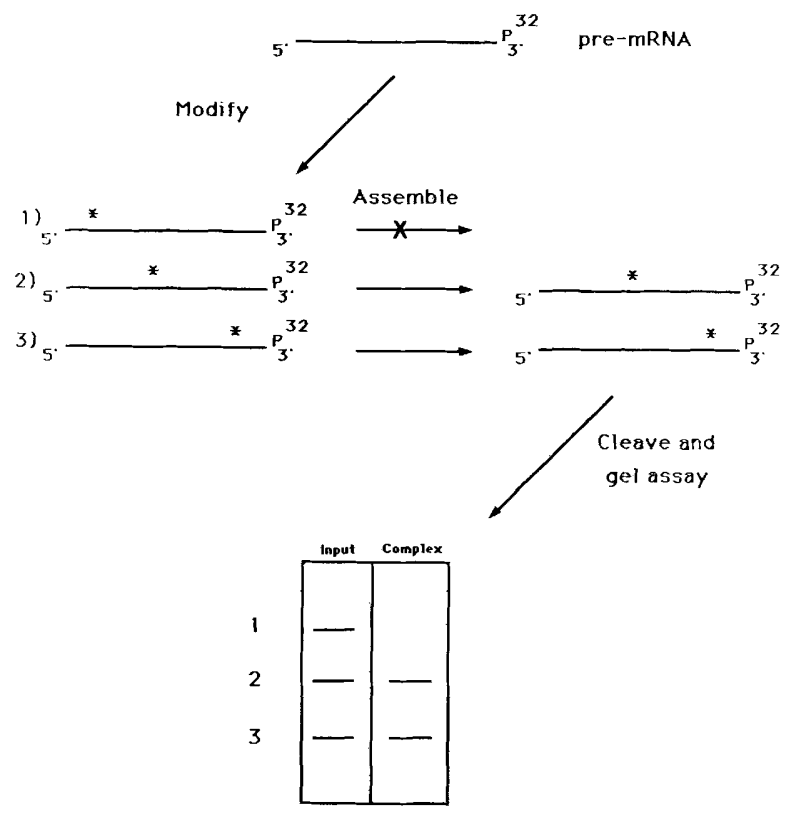

Figure 1. Outline of the chemical modification/interference assay. Pre-mRNA lesions (*) that inhibit spliceosome assembly are underrepresented in the pre-mRNA derived from one or more of the splicing complexes (Complex) relative to the input pre-mRNA (Input). See text for details. yeast whole-cell extract under standard splicing conditions (Lin et al. 1985). After 1 to $20 \mathrm{~min}$ of incubation (to maximize the yield of complexes III and I or complex II, respectivelyl, the splicing complexes were separated on non-denaturing polyacrylamide gels. The pre-mRNA was recovered from the individual splicing complexes, and the positions of the modifications were determined by subjecting the RNA to strand cleavage. Modified nucleotides present in reduced amount in the individual spliceosome complexes relative to those present in the substrate (input RNA) and in the pre-mRNA that fails to undergo assembly (the unassembled pool) were scored as inhibitory to spliceosome assembly, i.e., to complex I, II, or III formation (see Discussion for interpretation).

\section{Interference data}

Most nucleotides within the pre-mRNA could be modified without detectably interfering with spliceosome assembly (Fig. 2), i.e., the great majority of the cleavage sites in the starting pre-mRNA and in the unassembled pre-mRNA were also present in the RNA isolated from the spliceosome complexes. There were, however, some exceptions. In the $5^{\prime}$ splice site region, modifications at intron positions 2-6 (numbered from the first nucleotide of the intron, see Fig. 4) were reduced (position 5) or virtually absent (positions $2-4,6$ ) in pre-mRNA isolated from the initial form of the spliceosome (complex III). These lesions were further reduced (position 5) or unchanged (positions 2-4, 6) in pre-mRNA from the next step in the assembly pathway, complex I. Although ineffective in inhibiting complex III formation, modification of intron position 1 and the last nucleotide of exon $I / G$, position -1) inhibited complex I formation. Surprisingly, RNA modified at position -1 land, to a lesser extent, that modified at position -2) was enriched in complex III relative to the starting pre-mRNA or to the unassembled pool. We interpret this observation to indicate that modifications at these positions preferentially inhibit the progression from complex III to complex I, resulting in an increase in complex III pre-mRNA containing these lesions. The interference pattern of the $5^{\prime}$ splice site region from complex II-derived pre-mRNA was similar to that from complex I, with perhaps a slight additional increase in the exclusion of position 2 modifications.

The modified purines of the TACTAAC box also inhibited complex formation, although to varying extents (Fig. 2b). All three adenosines within the TACTAAC box were strongly inhibited from entering complex III [compare $\mathrm{A}+\mathrm{G}$ lanes $\mathrm{P}$ and $\mathrm{III}$; note that relative to the input RNA (lane P), the unassembled pool (lane U) contains more nonspecifically degraded RNA and therefore underrepresents the longer RNA species]. Modification of the adenosine located directly $3^{\prime}$ to the TACTAAC box (position 81) resulted in a slight underrepresentation of this lesion from complex III pre-mRNA but much greater exclusion from complexes I and II. Interestingly, a modified adenosine located 4 nucleotides $5^{\prime}$ to the TACTAAC box (position 70) had a minor but reproduc- 


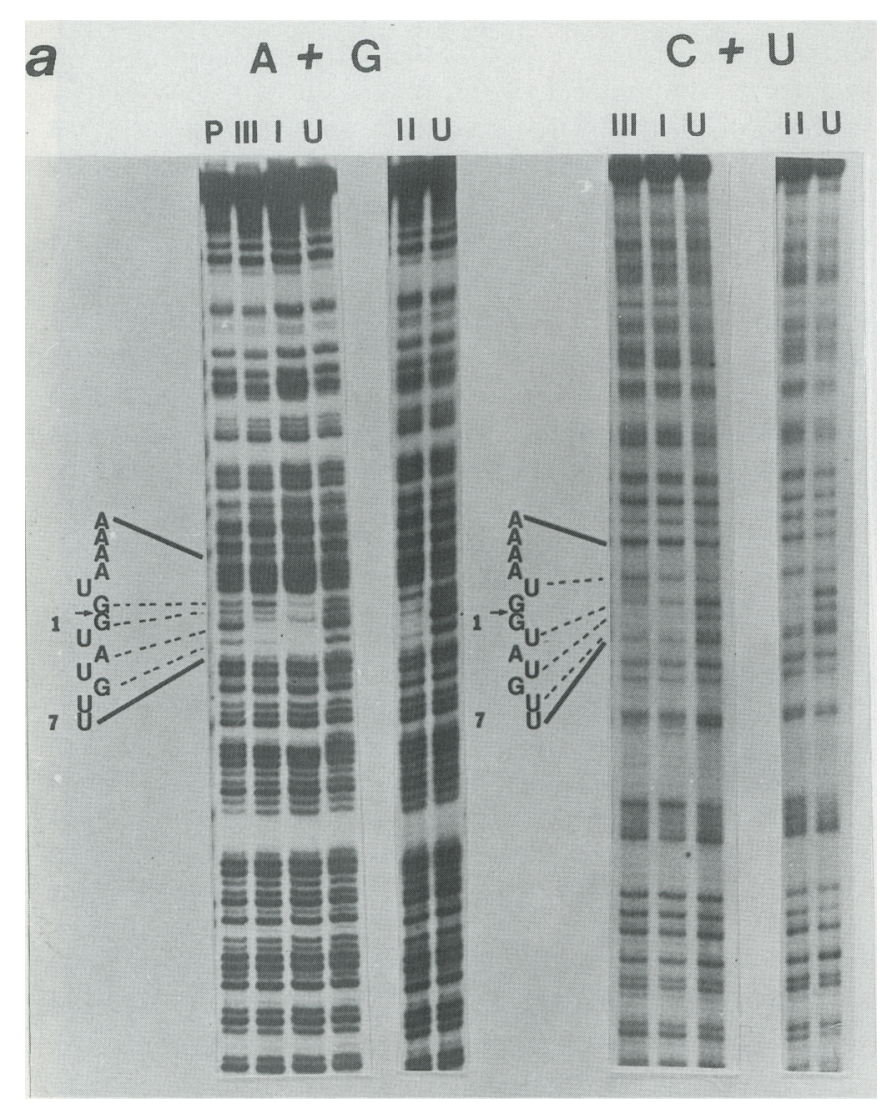

Figure 2. Modification/interference of spliceosome complex assembly. In vitro synthesized, chemically modified premRNAs were used as substrates for spliceosome assembly. The pre-mRNA was recovered from the complexes, and cleavage was induced at the sites of modification. Interfering modifications are indicated by the broken lines between the partial nucleotide sequence (bounded by solid lines) and the corresponding cleavage product. The arrows indicate the exon $\mathrm{I} / \mathrm{in}$ tron borders; the numbers refer to the corresponding intron positions. Lanes: $(A+G)$, DEPC reactions; $(C+U)$ hydrazine reactons; $(P)$ input pre-RNA; $(I I I)$ complex III pre-mRNA; $\{I\rangle$ complex I pre-mRNA; $(I I)$ complex II pre-mRNA; $(U)$ unassembled pre-mRNA. $(a)$ Cleavage pattern surrounding the $5^{\prime}$ splice site. Relative to complexes III and I $(C+U$ lanes $I I I$ and $I)$ complex II $(C+U$ lane $I I)$ pre-mRNA contains somewhat more of the position 6 modification. The extended incubation time required for maximum assembly of complex II (20 min versus 1 min for complexes III and I) likely allows the slow accumulation of RNA containing these lesions. $|b|$ Cleavage pattern surrounding the TACTAAC box. $(c)$ Cleavage pattern surrounding the $3^{\prime}$ splice site.

ible effect on complex formation (compare $A+G$, lanes $\mathrm{P}$ and $\mathrm{III})$, yet lesions in the intervening nucleotides (71-73) had no effect.

The pyrimidine lesions of the TACTAAC box were likewise inhibitory, although less so to complex III and I formation than to complex II formation ( $\mathrm{C}+\mathrm{U}$ lanes). The degree to which these modified nucleotides were excluded from the two early complexes was somewhat
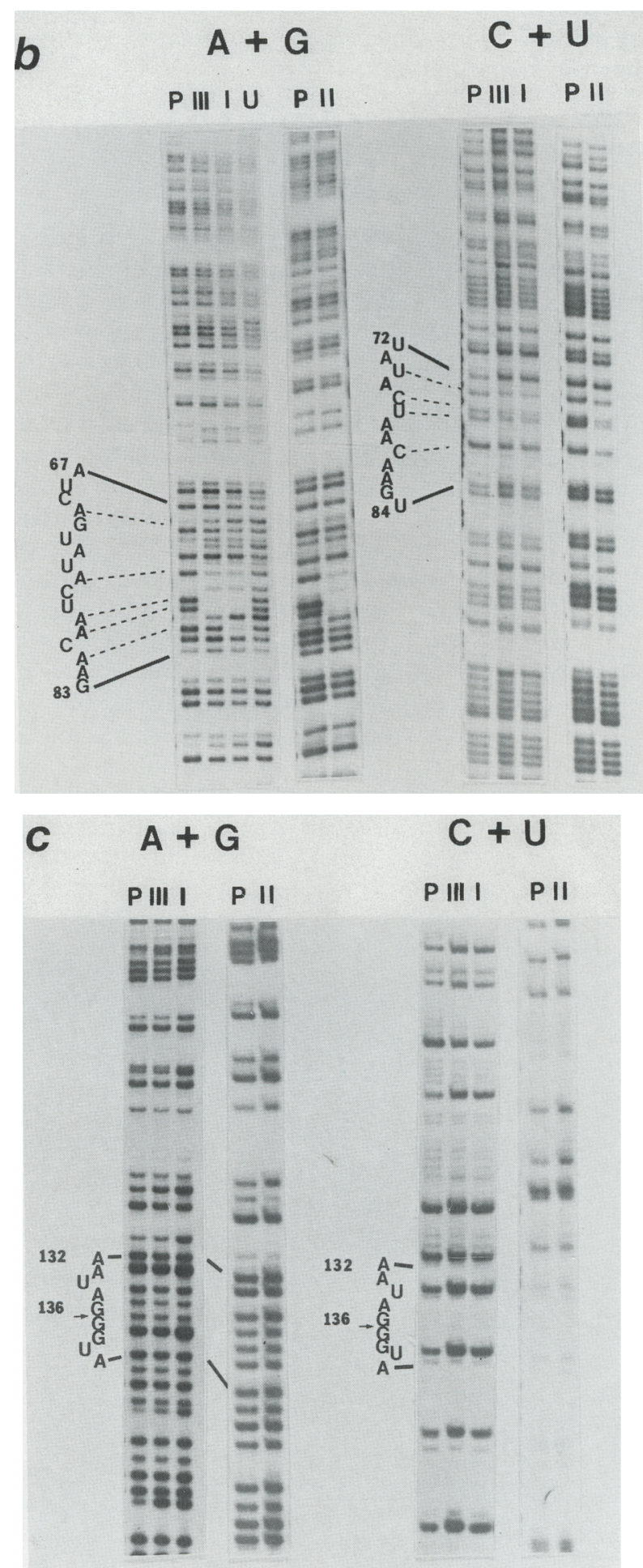

variable, ranging from the small, approximately twofold effect shown here to fourfold in other experiments. Nevertheless, in a given experiment the extent of modified pyrimidine exclusion was always less for the TACTAAC box than for the $5^{\prime}$ splice site (compare Fig. 2a,b). In contrast to the enhancement of the two terminal nucleotides of exon I in complex-III-derived pre-mRNA, the apparent enrichment of pyrimidine positions /such as 77 
and 80 ) in the complex III and complex I samples of the purine modification reactions (Fig. 2b, A + G lanes P, III, and I) is an artifact resulting from the isolation procedure. These aberrant cleavage sites are likewise present in the unassembled RNA pool (A+G lane $U)$.

Interestingly, modifications of the residues between the TACTAAC box and the $3^{\prime}$ splice site, including the $3^{\prime}$ splice site, had no reproducible effects on complex formation (Fig. 2c). This observation is consistent with our previous experiments that indicated that the rate of yeast spliceosome formation was independent of the presence of a 3' splice site (Rymond et al. 1987).

The effect of modified nucleotides on splicing was also examined (Fig. 3). The modification pattern of the lariat intermediate, debranched prior to RNA cleavage, was very similar to that of the pre-mRNA derived from complex II (compare, for example, Fig. 3a, lane 2 with Fig. $2 b, A+G, I I)$, although the extent of interference by certain lesions was somewhat stronger in the case of the lariat intermediate (note for instance the nearly complete exclusion of the adenosine at position 81). The modification pattern of the mRNA was difficult to compare with the input pre-mRNA, due to the discontinuity encountered upstream of the $3^{\prime}$ splice site (Fig. 3b). However, it was obvious that the modified $5^{\prime}$ terminal nucleotide of exon II (position +1 ) was absent from the mRNA. Likewise position +2 was somewhat reduced. Although positions farther downstream in exon II appear

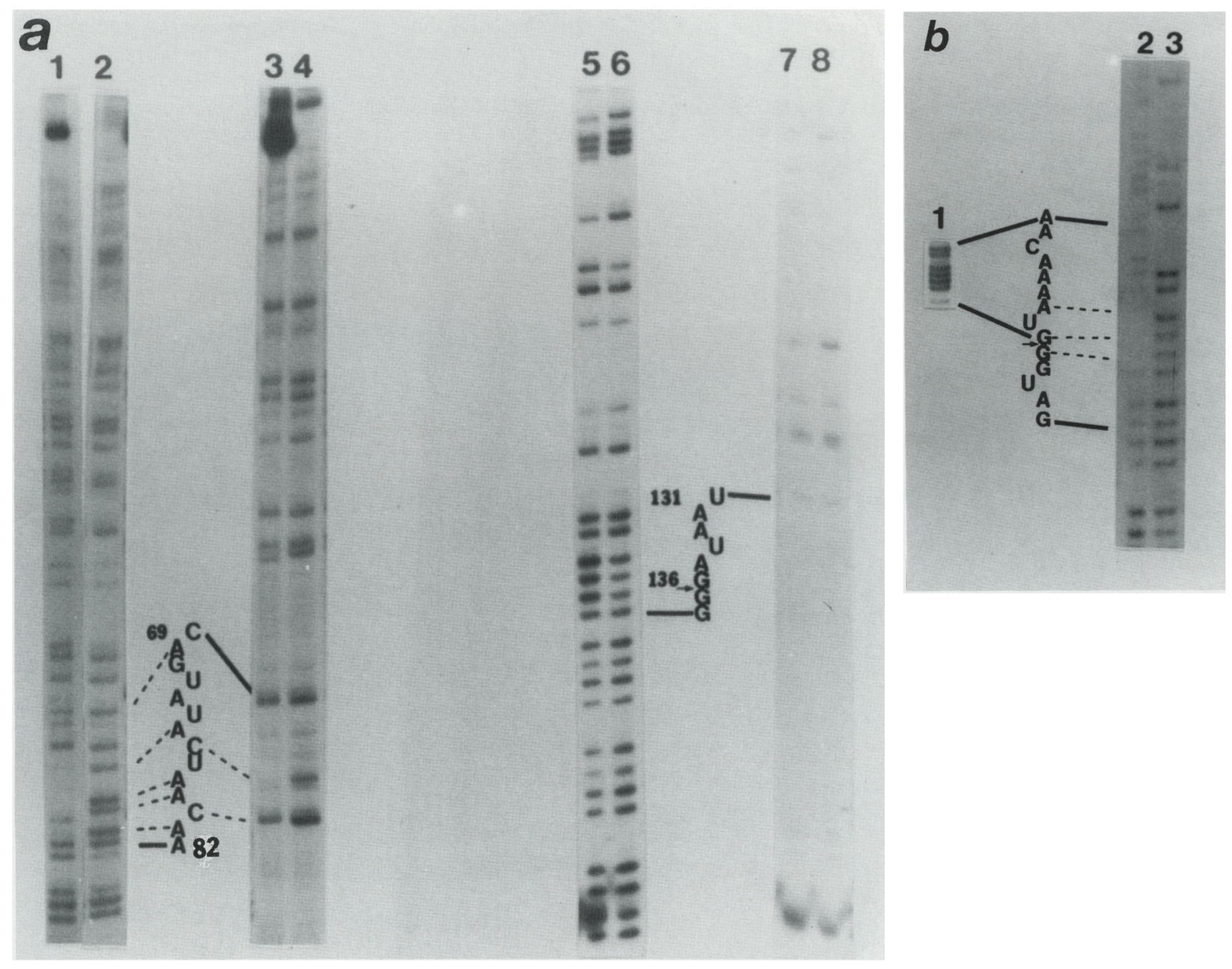

Figure 3. Modification/interference of splicing. $(a)$ The interference of modified nucleotides on lariat formation. Purine lesions $($ lanes $1,2,5$, and 6) and pyrimidine lesions (lanes $3,4,7$, and 81 present in the pre-mRNA (lanes 2, 4, 6, and 8) are compared with those present in the lariat intermediate (lanes 1,3,5, and 7). Lanes 1-4 focus on the nucleotides between the $5^{\prime}$ splice site and the TACTAAC box, and lanes 5-8 focus on the nucleotides surrounding the $3^{\prime}$ splice site. The numbers refer to the corresponding intron positions, and the arrow refers to the intron/exon II border. The uracil modifications are greatly underrepresented in this experiment; most of the bands in the pyrimidine lanes therefore represent cytosines. $(b)$ The interference of modified nucleotides on mRNA formation. Diethyl pyrocarbonate lesions within exon II of the pre-mRNA (lane 3, below the arrow) and exon I (lane 1) are compared with those within the mRNA (lane 2). The positions of affected nucleotides are indicated by the broken lines to the right of the partial mRNA sequence. The arrow indicates the exon I/exon II boundary. Nucleotides in exon I and exon II are numbered from this boundary and are refered to in the text as - and + positions, respectively. 
reduced (Fig. 3b), this effect was not reproducible (data not shown). Because modification of the position +1 nucleotide had no effect on complex formation nor on lariat-intermediate formation, it must specifically inhibit the second step of splicing.

A similar comparison was made between the exon I modification patterns of the pre-mRNA and mRNA. The four adjacent adenosines upstream of the $5^{\prime}$ splice site (positions -3 to -6 ) were modified with equal efficiency in the pre-mRNA (Fig. $3 b$, lane 1 ), yet the -3 adenosine was underrepresented in the mRNA (lane 2). Likewise, the uracil lesion at position -2 (data not shown) and the guanosine lesion at position -1 were suppressed in the mRNA. Because this technique could not examine the effect of these modified exon I nucleotides on the lariat intermediate, we were not able to determine if these lesions inhibited only the first step of splicing, only the second step, or both the first and second steps. In any case, modifications at these positions have a much more severe effect on mRNA formation than on complex formation /compare Fig. 2a and 3b). A compilation of the modification/interference data, representing several rounds of experimentation, is summarized in Figure 4 (see legend).

\section{Correspondence of chemical and mutational effects on} assembly

The data obtained using chemical modificaiton are generally in good agreement with those previously obtained with point mutants (see Discussion). However, most of the assays performed to date on mutant substrates did not distinguish between the various stages of spliceosome assembly. To test the predictive value of the modification/interference assay, we assayed several mutant pre-mRNAs for their effects on spliceosome assembly and splicing (Fig. 5a and 5b, respectively). As predicted from the modification data, point mutations at position 2 of the $5^{\prime}$ splice site (Fig. 5a; lanes $4-12$ ) or at positions 76 and 79 of the TACTAAC box (Fig. 5a; lanes 16-18 and 22-24, respectively) had marked effects on early spliceosome assembly (complex III formation). The extent of inhibition, however, varied as a function of the nucleotide change. A transversion at position 2 ( $U$ to $A$ or $U$ to $G$ ) resulted in virtually complete inhibition (Fig. $5 a$; lanes $7-9$ and $10-12$ ), while a transition (a $U$ to $C$ change) had a milder effect (Fig. 5a; lanes 4-6).

Modification of position 5 of the $5^{\prime}$ splice site modestly inhibited complex III formation $(\sim 2-3$ fold), yet virtually abolished complex I formation (Fig. 2a). A gua-
Figure 4. Summary of modification/interference data. These histograms illustrate the inhibitory effects of certain exon and intron sequences on spliceosome complex formation $(I I I, I$, and $I I)$, lariat formation $(L)$, and mRNA formation $(M)$. The data presented represent average values obtained from several repetitions (between 2 and 5 ) of the modification/ interference assay on complexes III and I, complex II, lariat intermediate and mRNA. Where no inhibition (or enhancement) was reproducibly visible by eye, the percent of recovery was set at 100 . In the other cases, the average percent of recovery was calculated from scans of autoradiograms and then set at the closest value, $150,125,75,50,25$, or 10 . The figure was designed to present a qualitative summary of the total modification pattern. The terminal two nucleotides of exon I in complex III exceed the $100 \%$ value, presumably due to inhibition of the complex III $\rightarrow$ I transition (see text for details). The numbers under the partial nucleotide sequence represent the corresponding intron position. Overlined from left to right are the $5^{\prime}$ splice site, TACTAAC box, and 3' splice site consensus sequences. Exon I and exon II nucleotides are numbered from the intron/ exon borders with negative $(-)$ and positive integers $(+1$, respectively.

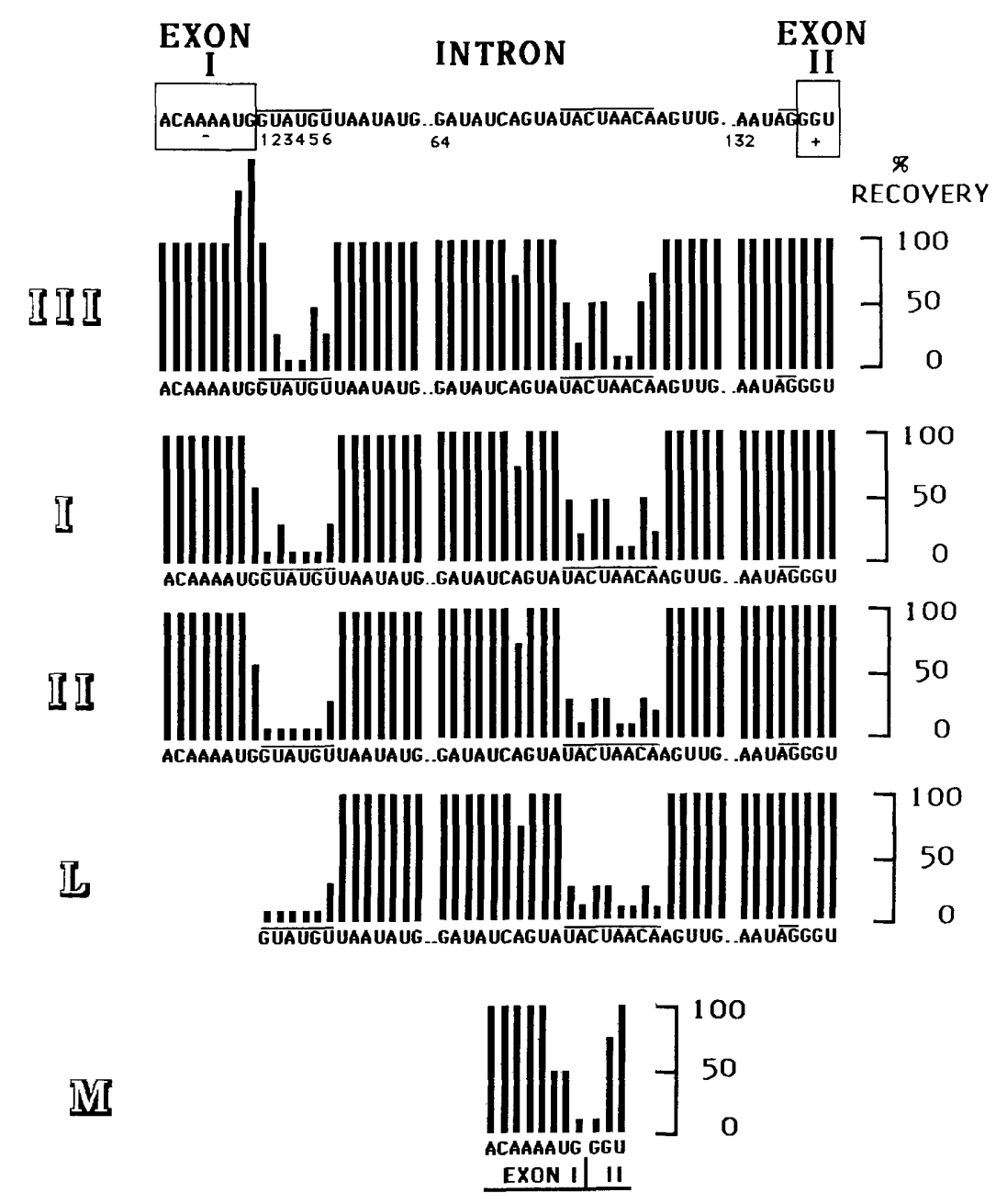



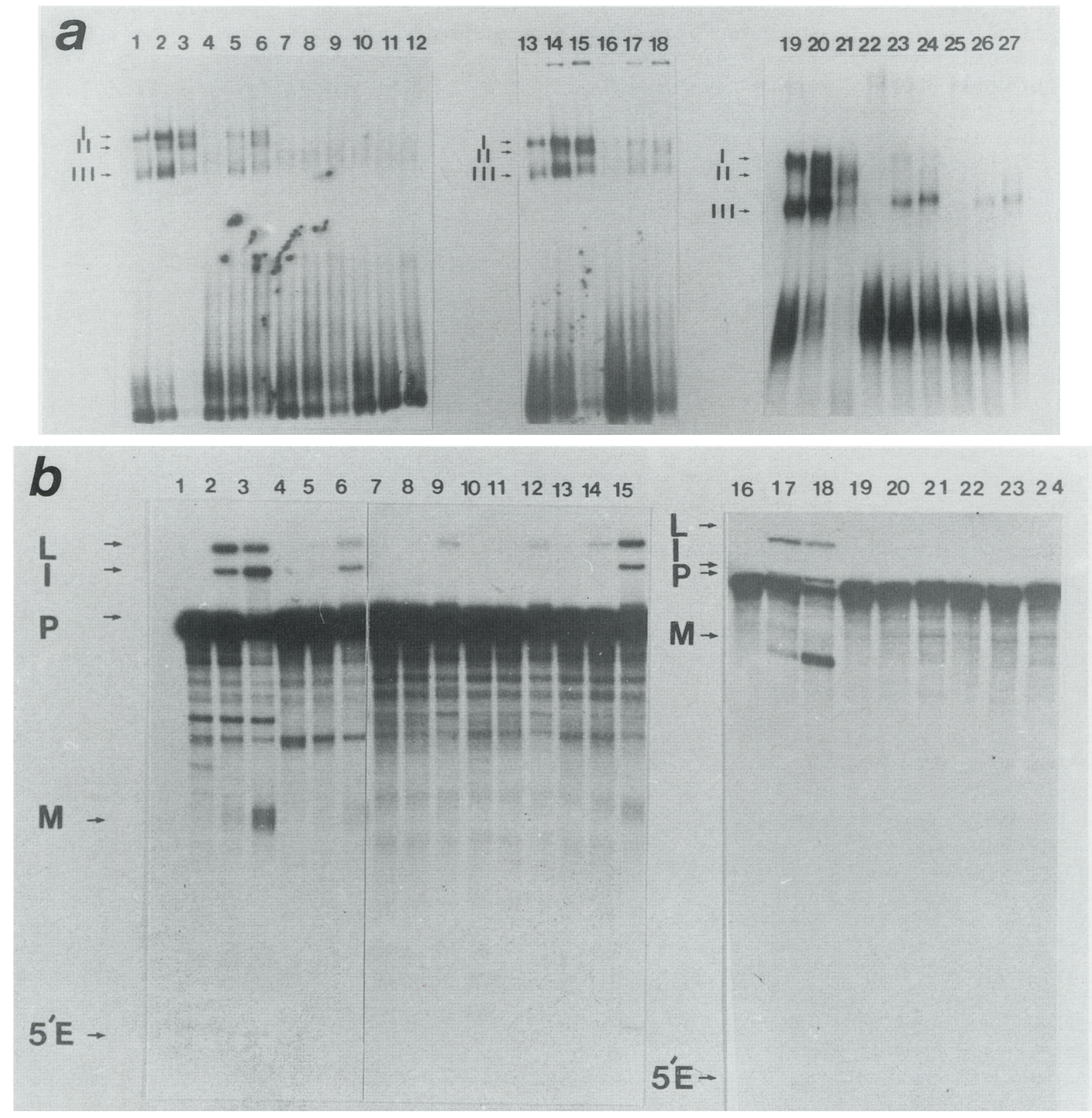

Figure 5. Time course of spliceosome formation and splicing of wild-type and mutant pre-mRNAs. $(a)$ Uniformly labeled splicing substrates were incubated for 1,5 , and $20 \mathrm{~min}$ in the yeast whole-cell extract, and the spliceosome complexes formed (I, II, and III) were resolved on native polyacrylamide gels. Wild-type transcripts (lanes 1-3, 13-15, and 19-21); intron position 2 mutants (U $\rightarrow \mathrm{C}$, lanes 4-6; $\mathrm{U} \rightarrow \mathrm{A}$, lanes 7-9; $\mathrm{U} \rightarrow \mathrm{G}$, lanes 10-12; the small amount of RNA present between complexes III and II in lanes 7-12 represents a gel artifact associated with the competitor RNA used to resolve the gel complexes [see Materials and methods]; intron position 76 mutant $(\mathrm{C} \rightarrow \mathrm{U}$, lanes 16-18), intron position 79 mutant $\mid \mathrm{A} \rightarrow \mathrm{C}$, lanes 22-24); intron position 5 mutant $(\mathrm{G} \rightarrow \mathrm{A}$, lanes 25-27). (b) Splicing analysis of wild-type and mutant pre-mRNAs. Splicing substrates were incubated in the whole cell extract for 1, 5, and $20 \mathrm{~min}$ and then were deproteinated and analyzed on denaturing $12 \%$ (lanes 1-15) or 5\% (lanes 16-24) gels. Wild-type transcripts (lanes 1-3, 16-18); position 76 mutant $(\mathrm{C} \rightarrow \mathrm{U}$, lanes 4-6); position 2 mutants $(U \rightarrow G$, lanes $7-9 ; \mathrm{U} \rightarrow \mathrm{A}$, lanes $10-12$; $U \rightarrow C$, lanes 13-15); position 79 mutant $(A \rightarrow C$, lanes 19-21); position 5 mutant $(G \rightarrow A$, lanes 22-24). Symbols: (L) lariat intermediate; (I) excised intron; (P) pre-mRNA; $(\mathrm{M})$ mRNA; $\left(5^{\prime} \mathrm{E}\right)$ exon $\mathrm{I}$.

nosine to adenosine transition at this position also affected both steps in spliceosome assembly (Fig. 5a, lanes 25-27). Complex III accumulated slowly, indicating that this change caused a considerable defect in the initial phases of assembly. Only a low level of complex I was detected. At late times complex II was clearly visible, suggesting that the small amount of complex I formed was efficiently converted into complex II.

The splicing defects of the mutant pre-mRNA transcripts parallel their assembly defects (Fig. 5b). The for- 
mation of splicing intermediates and products was decreased and delayed. As small amounts of both the lariat intermediate and the excised intron product were detected with almost every mutant transcript (Fig. 5b and data not shown) [the exception being the branch point mutant (lanes 19-21) which is very inefficiently but properly spliced in vivo (Jacquier and Rosbash 1986)], we conclude that the inefficient splicing of the mutant transcripts is a direct consequence of their poor spliceosome assembly.

\section{Identifying additional sites of interaction}

The data presented above demonstrate that splicesome assembly is most sensitive to modificaitons of the $5^{\prime}$ splice site and TACTAAC box. Presumably, this result reflects the primary roles these nucleotides play in the assembly process. To enhance the sensitivity of the assembly/interference assay and perhaps uncover additional nucleotides that modulate the efficiency of spliceosome formation, we used a mutant pre-mRNA transcript $(\mathrm{C} \rightarrow \mathrm{U}$ at position 76$)$ as a substrate for chemical modification and spliceosome assembly (Fig. 6). Although the background was somewhat higher (due in part to the comparatively low yield of splicing complex obtained using mutant substrates|, complex III assembly was sensitive to each of the lesions that inhibited the assembly of the wild-type transcript. Furthermore, modification of adenosines 67 and 70 (upstream of the TACTAAC box) and pyrimidines 68 and 69 (data not shown) inhibited stable complex formation. Nucleotides between position 70 and the TACTAAC box lor nucleotides elsewhere in the intron) showed no increased sensitivity to modification relative to a wild-type substrate. While modification of position 70 had been observed to decrease the assembly of the wild type transcript (Fig. $2 \mathrm{~b}$ ), the magnitude of this effect was greatly enhanced using the mutant pre-mRNA. This observation reinforces the suggestion that nucleotides near to and upstream of the TACTAAC box affect early complex formation. To verify that nucleotides 67 and 70 are indeed relevant to the assembly process, $\mathrm{A} \rightarrow \mathrm{C}$ transversions

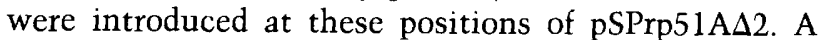
change at either position 67 or 70 resulted in a small but reproducible decrease in complex III formation (Fig. 7), suggesting a two- to threefold decrease in the initial rate of spliceosome assembly (splicing products were assayed only after $20 \mathrm{~min}$ of incubation and no effect of these mutations was observed; data not shown). As a control, position 73 was similarly changed $(\mathrm{A} \rightarrow \mathrm{C})$ with little or no effect on complex formation, in agreement with the modification/interference data shown above.

\section{Discussion}

Nucleic acid modifying agents have long been used as probes of nucleic acid structure and for the analysis of protein/nucleic acid interactions /Siebenlist and Gilbert 1980; Peattie and Herr 1981; and see references in Noller 1984). The chemical modification/interference assay de- scribed in this paper represents a logical yet simple extension of this technology. By selecting among a large, competing pool of randomly modified RNAs, the splicing factors effectively rank the effect of individual pre-mRNA lesions. At least three criteria must be met for the general application of this methodology. First, the RNA must be available in an amount and purity sufficient for RNA sequencing. Approximately 200 nucleotides from the labeled end of an RNA can be conveniently assayed. Second, the assembled state or products must be separable from the initial state of the RNA. Third, the reaction in question must be sensitive to single nitrogen base lesions within the RNA molecule.

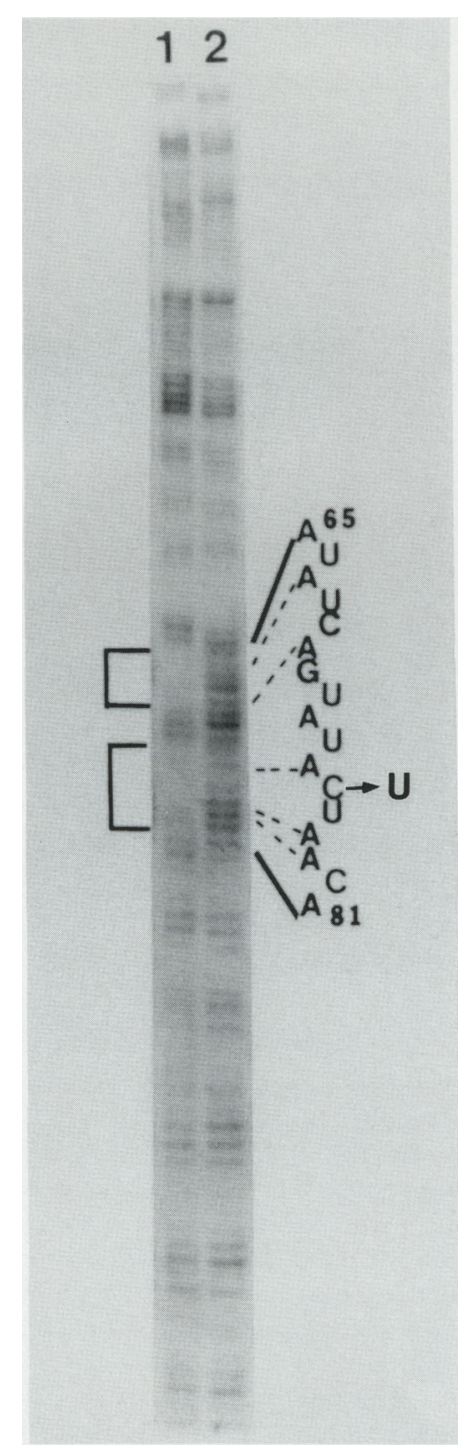

Figure 6. Modification/interference of assembly of mutant pre-mRNA. DEPC-modified mutant RNA (position 76, C $\rightarrow \mathrm{U}$ ) was used as a substrate for spliceosome assembly. Complex IIIderived RNA (lane 1) is compared with the unassembled premRNA (lane 2). The affected regions are indicated by the boxes to the right of lane 1 , and the individual affected nucleotides are indicated by the broken lines to the right of lane 2 . 


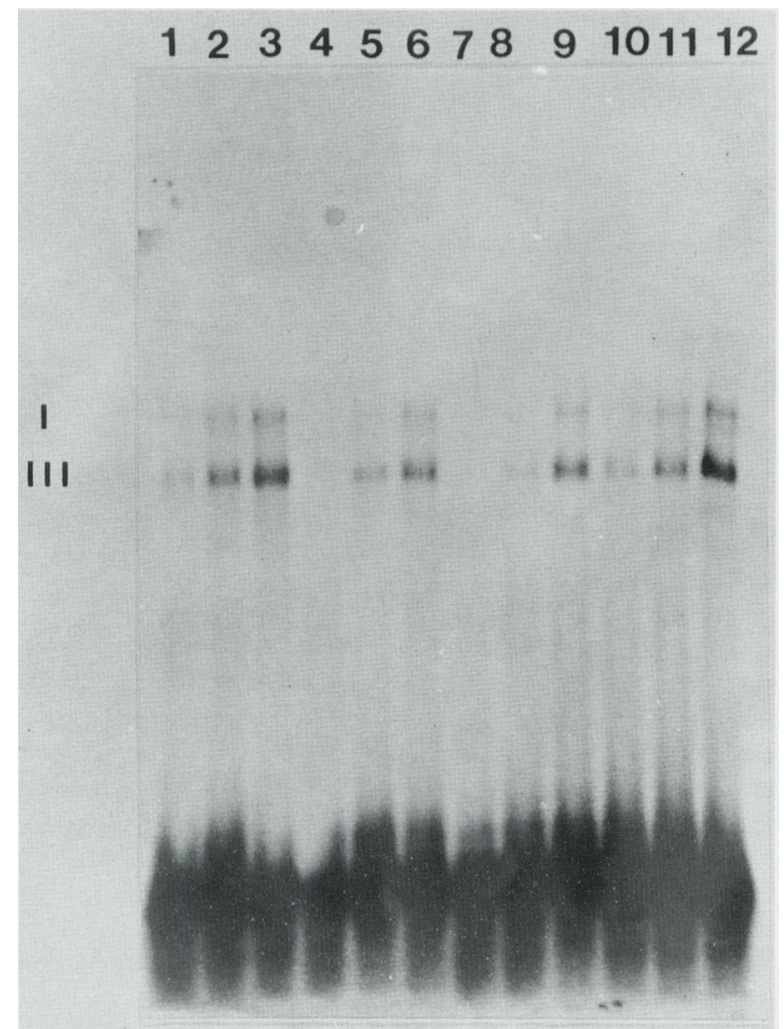

Figure 7. Kinetics of spliceosome formation on pre-mRNAs with mutations upstream of the TACTAAC box. The early $\{0.5$, $1.0,2.0 \mathrm{~min}$ ) assembly of spliceosomes on a wild-type transcript (lanes 1-3) was compared to the assembly of mutant transcripts: $\mathrm{A} \rightarrow \mathrm{C}$ of position 67 (lanes $4-6$ ); $\mathrm{A} \rightarrow \mathrm{C}$ of position 70 (lanes 7-9); and $\mathrm{A} \rightarrow \mathrm{C}$ of position 73 (lanes 10-12).

As this work was in progress we learned that Conway and Wickens (1987) have independently established and applied this experimental approach to the study of in vitro polyadenylation. The success of this methodology in these two very different experimental systems suggests that it will be widely applicable to the study of RNA/RNA and RNA/protein interactions.

\section{Pre-mRNA sequence requirements for stable complex formation}

The earliest detectable form of the yeast spliceosome (complex III) contains U2 small nuclear (sn) RNA (snR20; Ares 1986) and likely U1 snRNA (snR19; Kretzner et al. 1987; Siliciano et al. 1987b; Pikielny et al. 1986; Rymond et al. 1987; Kretzner et al. 1987). Base pairing between $\mathrm{U} 2$ and the TACTAAC box region of yeast introns facilitates splicing (Parker et al. 1987). Similarly, base pairing between $U 1$ and the 5 ' splice site of pre-mRNAs is required for both metazoan and yeast pre-mRNA splicing (Zhuang and Weiner 1986; B. Seraphin and $M$. Rosbash, in prep.). These data suggest that the snRNA/pre-mRNA base pairing interactions may be required for the initial stage of spliceosome assembly (complex III formation). Consistent with this notion, modification of several positions in the $5^{\prime}$ splice site and the TACTAAC box significantly inhibit complex III formation. Surprisingly, however, modification of other positions within these regions (the $3^{\prime}$ terminal G of exon I, the first and fifth $\mathrm{G}$ of the $5^{\prime}$ splice site, the last $\mathrm{A}$ of the TACTAACA consensus, and the pyrimidines in the TACTAAC box) have no effect or are only modestly inhibitory. This observation may reflect the fact that only a subset of the nucleotides within the two consensus sequences are important for complex III formation. If this inhibition pattern reflects RNA/RNA interactions (snRNA/pre-mRNA), it may suggest that only a subset of the potential pairings take place early in assembly. Alternatively, the pattern may reflect the critical nucleotides involved in protein/RNA contacts (e.g., snRNP protein/pre-mRNA contacts) that are required for complex III formation and that precede snRNA/pre-mRNA base pairing. As recent data from this lab indicate that U1 snRNA/pre-mRNA base pairing (including position 5 of the $5^{\prime}$ splice site) is required for complex III formation (B. Seraphin and M. Rosbash, in prep.), the relatively weak effect of the three modified guanosine nucleotides in the $5^{\prime}$ splice site on complex III formation more likely reflects the nature of the nucleotide lesion rather than a lack of base pairing with Ul prior to complex III formation. Carbethoxylation of N-7 does not directly affect an atom involved in hydrogen bonding. Although RNA secondary structure can block carbethoxylation of $\mathrm{N}^{7}$ (Inoue and Cech 1985), the structural perturbation of such a lesion within an RNA may be insufficient to prevent base pairing.

Hydrazine lesions (removal of the pyrimidine base) of the $5^{\prime}$ splice site are strong inhibitors of complex III formation, while hydrazine lesions within the TACTAAC box are relatively weak inhibitors of complex III formation. The different response to hydrazine treatment may reflect the fact that $\mathrm{U} 1$ pairing with the $5^{\prime}$ splice site is important for substrate recognition and early complex formation while $\mathrm{U} 2$ pairing with the branch point region is not, a suggestion for which there is some experimental support from metazoa (Kramer 1987; Zillmann et al. 1988).

Modification of consensus sequence nucleotides presumed to be unpaired with their associating snRNAs (the uracil at position 4 of the $5^{\prime}$ splice site and the branch point A) markedly inhibits complex formation. This may result in part from the fact that the chemical lesions impose a more severe structural perturbation than a normal, unpaired nucleotide. Nevertheless, the sequence conservation of these positions in yeast introns (position 4 of the $5^{\prime}$ splice site is almost always a uracil and always a pyrimidine, and the branch point is always an adenosine) and the strong phenotypes of mutations at these positions (Langford et al. 1984; Newman et al. 1985; Fouser and Friesen 1986; Jacquier and Rosbash 1986; B. Seraphin and M. Rosbash, in prep.) indicate a role for these unpaired nucleotides in spliceosome assembly, perhaps involving protein/pre-mRNA interaction.

Modification of an adenosine 4 nucleotides upstream of the TACTAAC box (position 70) caused a two- to 
threefold decrease in complex III formation. With a mutant transcript, the magnitude of this effect was enhanced and the sensitive region expanded to include three adjacent nucleotides (intron positions 67-70). $\mathrm{A} \rightarrow \mathrm{C}$ transversions of positions 67 and 70 likewise inhibit complex III formation. Taken together, these results suggest that the region upstream of but perhaps not contiguous with the TACTAAC box modulates the rate of stable complex formation. We have previously demonstrated that the sequence context bordering the intron consensus sequences can alter the efficiency of splicing (Pikielny and Rosbash 1985) and that the sequences upstream of the TACTAAC box appear to interact with splicing factors (Rymond and Rosbash 1986). It is interesting to note that this upstream sequence falls within a region that closely resembles the TACTAAC box (see Fig. 81. A single nucleotide change in this region that creates a sequence more similar to the TACTAAC box partially suppresses the splicing defect of a branch point mutation, allowing for greater utilization of the authentic (yet mutant) TACTAAC box (Jacquier and Rosbash 1986). An imperfect branch point sequence is also present downstream of the RP51A TACTAAC box (Pikielny et al. 1983) and in several other yeast genes (Cellini et al. 1986). The yeast actin gene has an imperfect and upstream TACTAAC box which can be 'activated' by deletion of the authentic TACTAAC box /Cellini et al. 1986). Our results suggest that the upstream TACTAAC-box-like sequence modulates the efficiency of the splicing by facilitating spliceosome assembly. Since there is no indication that the degenerate TACTAAC box itself is used as a site of branch point formation, its influence is likely indirect. Splicing factors bound at this sequence might, through cooperative interactions, stabilize similar interactions at the authentic TACTAAC box. Alternatively, as has been suggested for the yeast actin gene (Cellini et al. 1986), the TACTAAC box-like sequence may act as a sink for limiting splicing factors; factors initially bound at this sequence might subsequently migrate along the RNA chain until the authentic TACTAAC box is reached. In either case, the local environment of the yeast branch point appears to be more ordered than originally envisioned. Perhaps the "alternative" branch points of metazoan transcripts (Ruskin et al. 1985) are likewise repetitive sequence elements that serve to attract branch point binding splicing factors and that become utilized for lariat formation only after the loss of the primary branch point sequence.

\section{Pre-mRNA sequence requirements for spliceosome maturation and splicing}

Complex I is derived from complex III and contains snR7, snR14, and snR6 (the yeast equivalents of U5, U4, and U6, respectively; Patterson and Guthrie 1987; Siliciano et al. 1987a), in addition to U2 and probably U1, inherited from complex III (Pikielny et al. 1986; Cheng and Abelson 1987; Rymond et al. 1987). Relative to complex III formation, the pre-mRNA sequence requirements for complex I formation are much more stringent.

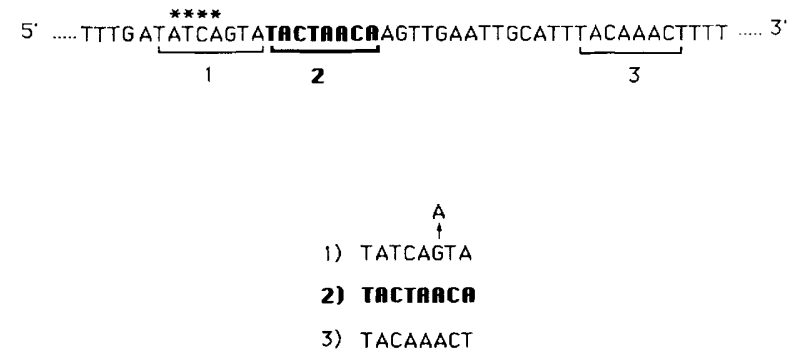

Figure 8. Partial nucleotide sequence surrounding the RP51A TACTAAC box. $(a)$ The TACTAAC box is indicated by boldface lettering. The positions upstream of the TACTAAC box that, when modified, inhibit the assembly of the position 76 mutant pre-mRNA are indicated by asterisks. Underlined are the nucleotides encompassing the TACTAAC box $\{2\}$ and the two flanking TACTAAC-box-like sequence elements ( 1 and 3 ). (b) Comparison of the TACTAAC box with elements 1 and 3 . The arrangement of purines and pyrimidines in element 1 is identical to the TACTAAC box and 4 of the 8 nucleotides are identical. The similarity increases to $5 / 9$ nucleotides if the adenosine $5^{\prime}$ to both sequences is included in the comparison. $A G \rightarrow A$ transition of element 1 (indicated by the arrow) increases this similarity to $6 / 9$ nucleotides. Element 3 fits the TACTAAC box in 6 of 8 positions. However, two of the nucleotide differences involve base pair transversions. These changes may inactivate element 3 and account for the lack of inhibition of modified nucleotides in this region of the intron.

Modification of any nucleotide of the $5^{\prime}$ splice site or TACTAAC box inhibits complex I formation, and several of the nucleotide lesions that inhibit complex III formation affect complex I formation more severely. The increased sensitivity of complex I to chemical lesions correlates with the increased resistance of the $5^{\prime}$ splice site to oligonucleotide-directed RNase $\mathrm{H}$ cleavage and with the increased sensitivity of complex I to certain pre-mRNA mutations (Pikielny et al. 1986; Rymond and Rosbash 1986 and unpubl.). These observations likely reflect the increased demands placed upon the 5' splice site and TACTAAC box sequences by the association of additional splicing factors (for instance, the snR6, snR7, and snR14 snRNPs). Conceivably, these demands stem from direct effects (i.e., binding of new factors to these consensus sequences or to snRNA/pre-mRNA base paired regions) or indirect effects (e.g., 'stress' on pre-existing interactions due to conformational changes within the spliceosome), imposed, for example, by the association of complex I splicing factors.

The complex I $\rightarrow$ complex II transition takes place during both yeast and mammalian spliceosome assembly and is characterized by the loss of U4 snRNA from the spliceosome (Pikielny et al. 1986; Konarska and Sharp 1987). Complex II appears to be the mature spliceosome and contains, in addition to pre-mRNA, the intermediates and products of the splicing reaction. Relative to complex-I-derived pre-mRNA, complex-II-derived pre-mRNA and lariat intermediate RNA were both somewhat more sensitive to chemical lesions at the $5^{\prime}$ splice site and TACTAAC box. These observations reinforce the three-step model of spliceosome formation 
(complex III $\rightarrow$ I $\rightarrow$ II) and suggest further that complex II assembly and the release of U4 snRNA are obligate steps in the splicing pathway. This increased sensitivity also indicates roles for the consensus sequence nucleotides in the later stages of spliceosome maturation and in step 1 of the splicing reaction. Analysis of the final exon-I-exon-II ligation product revealed that modification of the last three nucleotides of exon I and the first two nucleotides of exon II inhibited mRNA formation. The lesions in exon I may inhibit either step $1\left(5^{\prime}\right.$ splice site cleavage and lariat formation) or step $2 / 3^{\prime}$ splice site cleavage and exon ligation). However, the exon II sequence requirements must reflect a step 2 effect since modification of either of these two nucleotides had no effect on lariat intermediate formation. The presence of specific sequence requirements for step 2 in splicing is consistent with mutagenesis data (Newman et al. 1985; Fouser and Friesen 1986, 1987; Vijayraghavan et al. 1986).

\section{Conclusions}

The observed phenotypes of the purine and pyrimidine modifications give a much more detailed picture of premRNA sequence requirements for in vitro spliceosome assembly and in vitro splicing than was previously available from selected mutagenesis studies (Newman et al. 1985; Rymond and Rosbash 1985; Pikielny et al. 1986; Vijayraghavan et al. 1986; Rymond et al. 1987). A number of positions within the RP51A pre-mRNA have been identified that are required for efficient formation and maturation of yeast spliceosomes and for the twostep splicing reaction. These essential regions include, but are not limited to, the three defined intron consensus sequences, the $5^{\prime}$ splice site, the TACTAAC box, and the $3^{\prime}$ splice site. Within each of these conserved sequence elements, the contribution of individual nucleotides varies during assembly and splicing, suggesting multiple roles for these sequence landmarks in premRNA splicing.

\section{Materials and methods}

\section{Preparation of splicing extracts}

Yeast whole-cell extract was prepared from strain EJ101 $(\alpha$, trp1, pro1-126, prb1-112, pep4-3, pro1-126) essentially as previously described (Lin et al. 1985). Spheroplast formation was assayed spectrophotometrically by diluting $25 \mu$ l of the cell suspension in $1 \mathrm{ml}$ of $0.1 \%$ SDS and monitoring the absorbance at $600 \mathrm{~nm}$. After a $70 \%$ drop in absorbance was attained, the spheroplasts were diluted $1: 7.5$ in YMl sorbitol 11 g Bacto yeast extract, $2 \mathrm{~g}$ Bacto peptone, $1 \mathrm{~g}$ succinic acid, $0.6 \mathrm{~g}$ sodium hydroxide, $2 \mathrm{~g}$ dexgrose, and $18.22 \mathrm{~g}$ sorbitol per $100 \mathrm{ml}$ ) and incubated at $30^{\circ} \mathrm{C}$ with gentle shaking $(\sim 100 \mathrm{rpm})$ for $90 \mathrm{~min}$. The cells were collected by centrifugation $15000 \mathrm{rpm} 5 \mathrm{~min}$ in a Sorvall SS 34 rotor), washed twice with $30 \mathrm{ml}$ of cold $1 \mathrm{M}$ sorbitol, $10 \mathrm{~mm} \mathrm{MgCl}$, $3 \mathrm{~mm} \mathrm{DTT}, 50 \mathrm{~mm}$ Tris- $\mathrm{HCl}$ (pH 7.8) and then resuspended in $10 \mathrm{ml}$ of cold homogenization buffer [10 mM HEPES ( $\mathrm{pH} 7.0$ ), $1.5 \mathrm{~mm} \mathrm{MgCl}_{2}, 10 \mathrm{mM} \mathrm{KCl}, 0.5 \mathrm{~mm}$ DTT, $0.5 \mathrm{~mm}$ PMSF]. Cell lysis and all subsequent steps were carried out as originally described (Lin et al. 1985).

\section{Plasmids, RNA preparation}

Plasmids pSPrp51A and pSPrp51A $\Delta 2$ have been previously described (Pikielny and Rosbash 1986; Ruskin et al. 1986; Rymond et al. 1987). Mutant derivatives pSPrp51A(3'III), pSPrp51A $\left(5^{\prime} \mathrm{II}\right)$ were prepared by inserting the intron-containing HindIII-PvulI fragment of 3'III and 5'II (Jacquier et al. 1985), respectively, into vector pSP62. The intron position 2 mutants (GTATGT) and position 76 mutant (TACTAAC) were generated by oligonucleotide directed mutagenesis (Amersham in vitro mutagenesis system) of $\mathrm{pT} 7 \Delta 2$ (containing the HindIII$\mathrm{SacI}$ intron fragment of $\mathrm{p} \Delta 2$ (Pikielny et al. 1983) inserted into vector pTZ19U (US Biochemicals). In vitro transcriptions were performed as described (Ruskin et al. 1986) on plasmids digested with EcoRI (pSPrp51A, pSPrp51A(3'III) and pSPrp51A(5'II) or DdeI (pSPrp51A $\Delta 2$ and its mutant derivatives) using SP6 polymerase (Promega) or T7 polymerase (USB). The purification of full-length transcripts and the 3 'end-labeling of the RNA were performed as described (Pikielny and Rosbash 1986).

\section{Chemical modifications}

As described by Peattie (1979), diethyl pyrocarbonate (DEPC, Sigma) and hydrazine (Aldrich) were used to modify the purine and pyrimidine residues, respectively, of the yeast pre-mRNA transcripts. Approximately $1 \times 10^{7} \mathrm{cpm}$ of labeled pre-RNA and $5 \mu \mathrm{g}$ tRNA were used in each modification reaction. The times of incubation required for limited modification (less than one lesion per molecule) were: $\operatorname{DEPC}(A+G, 1 \mathrm{~min})$, hydrazine $(\mathrm{C}+\mathrm{U}, 15 \mathrm{~min} ; \mathrm{U}, 2 \mathrm{~min})$. To balance the relative abundance of uracil and cytosine lesions, equal amounts of hydrazine $\mathrm{C}+\mathrm{U}$ and $\mathrm{U}$ reactions were added to the pyrimidine modification/interference experiment. Strand cleavage was induced on the RNA recovered from each modification/interference experiment by the addition of $20 \mu \mathrm{l}$ of $1 \mathrm{M}$ analine (Aldrich), $0.6 \mathrm{M}$ sodium acetate $(\mathrm{pH} 4.0)$ and incubation at $60^{\circ} \mathrm{C}$ in the dark for 20 min. $n$-Butanol $(1.5 \mathrm{ml})$ was added to terminate this reaction, and the RNA was precipitated by centrifugation for $3 \mathrm{~min}$ in an Eppendorf microfuge. The supernatant was discarded, and the pellet was resuspended in $200 \mu \mathrm{l}$ of $1 \%$ SDS. The RNA was again precipitated with butanol and then washed with $1 \mathrm{ml}$ of $90 \%$ ethanol. The pellets were dried under vacuum and resuspended in $10 \mu \mathrm{l}$ of loading buffer $[8 \mathrm{M}$ urea, $20 \mathrm{~mm}$ Tris- $\mathrm{HCl}(\mathrm{pH}$ 7.4), $1 \mathrm{mM}$ EDTA, $0.05 \%$ bromphenol blue]. The samples were denatured at $90^{\circ} \mathrm{C}$ for 1 min prior to loading on a denaturing, $7 \%$ polyacrylamide sequencing gel. The relative intensities of the RNA cleavage products were determined using a BioRad model 620 video densitometer.

\section{Spliceosome assembly reactions, native polyacrylamide gels}

Approximately $2 \times 10^{6} \mathrm{cpm}$ of chemically modified RNA were added to each $50 \mu \mathrm{l}$ (final volume) spliceosome assembly reaction (Lin et al. 1985). After $1 \mathrm{~min}$ of incubation (for the isolation of complexes III and I) or $20 \mathrm{~min}$ of incubation (for the isolation of complex II) at $25^{\circ} \mathrm{C}$, the reaction was stopped by the addition of an equal volume of Q buffer (Pikielny and Rosbash 1986). Ten percent of the sample was deproteinated by proteinase $\mathrm{K}$ treatment followed by phenol extraction (Pikielny and Rosbash 1986). This RNA was precipitated with ethanol and used as the "input RNA" control in the cleavage reaction. To the remainder of the splicing reaction $100 \mu \mathrm{g}$ of total yeast RNA were added and the spliceosome complexes were resolved on a preparative $3 \%$ acrylamide $/ 0.5 \%$ agarose gel (Pikielny and Rosbash 1986). The labeled substrate RNA was electroblotted from the gel onto DEAE membrane, and then recovered from this mem- 
brane as described (Pikielny and Rosbash 1986). To isolate complex II pre-mRNA free of the other labeled RNAs found in this complex (lariat intermediate and mRNA molecules), the sample was further fractionated on a denaturing $5 \%$ polyacrylamide gel. The lariat intermediate and mRNA species were directly isolated from 20 -min splicing reactions by deproteination and fractionation on denaturing $5 \%$ polyacrylamide gels. Lariat intermediate molecules were linearized with $\mathrm{HeLa}$ cell debranching activity (Ruskin and Green 1985) prior to the strand cleavage reaction.

\section{Acknowledgments}

We wish to thank L. Conway and M. Wickens for communicating their results prior to publication; our colleagues P. Legrain, S. Fromherz, L. Kretzner, B. Seraphin, N. Abovich, and H. Colot for their helpful suggestions and criticisms while this work was in progress; and C. Pikielny and M. Green for their comments on this manuscript. This work was supported by NIH grant GM23549 to M.R. and an NIH postdoctoral fellowship to B.C.R.

\section{References}

Ares, M., Jr. 1986. U2 RNA from yeast is unexpectedly large and contains homology to vertebrate U4, U5, and U6 small nuclear RNAs. Cell 47: 49-59.

Brody, E. and J. Abelson. 1985. The 'spliceosome': Yeast premessenger RNA associates with a $40 \mathrm{~S}$ complex in a splicing-dependent reaction. Science 228: 963-967.

Cech, T.B. and B.L. Bass. 1986. Biological catalysis by RNA. Annu. Rev. Biochem. 55: 599-629

Cellini, A., R. Parker, J. McMahon, C. Guthrie, and J. Rossi. 1986. Activation of a cryptic TACTAAC box in the Saccharomyces cerevisiae actin intron. Mol. Cell. Biol. 6: 15711578.

Cheng, S.-C. and J. Abelson. 1987. Spliceosome assembly in yeast. Genes Dev. 1: 1014-1027.

Conway, L. and M. Wickens. 1987. Analysis of mRNA $3^{\prime}$ end formation by modification interference: the only modifications which prevent processing lie in AAUAAA and the poly(A) site. EMBO I. 6: 4177-4184.

Fouser, L.A. and J.D. Friesen. 1986. Mutations in a yeast intron demonstrate the importance of specific conserved nucleotides for the two stages of nuclear mRNA splicing. Cell 45: $81-93$.

__ 1987. The effects on mRNA splicing of mutations in the 3 ' region of the Saccharomyces cerevisiae actin intron. Mol. Cell. Biol. 7: 225-230.

Green, M.R. 1986. Pre-mRNA splicing. Annu. Rev. Genet. 20: $617-708$.

Inoue, T. and T.R. Cech. 1985. Secondary structure of the circular form of the Tetrahymena rRNA intervening sequence: A technique for RNA structure analysis using chemical probes and reverse transcriptase. Proc. Natl. Acad. Sci. 82: $648-652$.

Jacquier, A., J.R. Rodriguez, and M. Rosbash. 1985. A quantitative analysis of the effects of $5^{\prime}$ junction and TACTAAC box mutants and mutant combinations in yeast mRNA splicing. Cell 43: 423-430.

Jacquier, A. and M. Rosbash. 1986. RNA splicing and intron turnover are greatly diminished by a mutant yeast branch point. Proc. Natl. Acad. Sci. 83: 5835-5839.

Konarska, M.M. and P.A. Sharp. 1987. Interactions between small nuclear ribonucleoprotein particles in formation of spliceosomes. Cell 49: 763-774.
Kramer, A. 1987. Analysis of RNase-A-resistant regions of adenovirus 2 major late precursor-mRNA in splicing extracts reveals an ordered interaction of nuclear components with the substrate RNA. I. Mol. Biol. 196: 559-573.

Kretzner, L., B.C. Rymond, and M. Rosbash. 1987. S. cerevisiae U1 RNA is large and has limited primary sequence homology to metazoan Ul snRNA. Cell 50: 593--602.

Langford, C.J., F.J. Klinz, C. Donath, and D. Gallwitz. 1984. Point mutations identify the conserved, intron-contained TACTAAC box as an essential signal sequence in yeast. Cell 36: 645-653.

Lin, R.-J., A.J. Newman, S.-C. Cheng, and J. Abelson. 1985. Yeast mRNA splicing in vitro. I. Biol. Chem. 260: 1478014792.

Lin, R.-J., A.J. Lusting, and J. Abelson. 1987. Splicing of yeast nuclear pre-mRNAs in vitro requires a functional $40 \mathrm{~S}$ spliceosome and several extrinsic factors. Genes Dev. 1: 7-18.

Maniatis, T. and R. Reed. 1987. The role of small nuclear ribonucleoprotein particles in pre-mRNA splicing. Nature 325: 673-678.

Newman, A.J., R.-J. Lin, S.-C. Cheng, and J. Abelson. 1985. Molecular consequences of specific intron mutations on yeast mRNA splicing in vivo and in vitro. Cell 42: 335-344.

Noller, H.F. 1984. Structure of ribosomal RNA. Annu. Rev. Biochem. 53: 119-162.

Padgett, R.A., P.J. Grabowski, M.M. Konarska, S. Seiler, and P.A. Sharp. 1986. Splicing of messenger RNA precursors. Annu. Rev. Biochem. 55: 119-150.

Parker, R., P.G. Siliciano, and C. Guthrie. 1987. Recognition of the TACTAAC box during mRNA splicing in yeast involves base pairing to the U2-like snRNA. Cell 49: 229-239.

Patterson, B. and C. Guthrie. 1987. An essential yeast snRNA with a U5-like domain is required for splicing in vivo. Cell 49: $613-624$.

Peattie, D.A. 1979. Direct chemical method for sequencing RNA. Proc. Natl. Acad. Sci. 76: 1760-1764.

Peattie, D.A. and W. Herr. 1981. Chemical probing of the tRNA-ribosome complex. Proc. Natl. Acad. Sci. 78: 22732277.

Pikielny, C.W. and M. Rosbash. 1985. mRNA splicing efficiency in yeast and the contribution of non-conserved sequences. Cell 41: 119-126.

- 1986. Specific small nuclear RNAs are associated with yeast spliceosomes. Cell 45: 869-877.

Pikielny, C.W., J.L. Teem, and M. Rosbash. 1983. Evidence for the biochemical role of an internal sequence in yeast nuclear mRNA introns: Implications for U1 RNA and metazoan mRNA splicing. Cell 34: 395-403.

Pikielny, C.W., B.C. Rymond, and M. Rosbash. 1986. Electrophoresis of ribonucleoproteins reveals an ordered assembly pathway of yeast splicing complexes. Nature 324: 341-345.

Ruskin, B. and M.R. Green. 1985. An RNA processing activity that debranches RNA lariats. Science 229: 135-140.

Ruskin, B., J.M. Green, and M.R. Green. 1985. Cryptic branch point activation allows accurate in vitro splicing of human beta-globin intron mutants. Cell 41: 833-844.

Ruskin, B., C.W. Pikielny, M. Rosbash, and M.R. Green. 1986. Alternative branch points are selected during splicing of a yeast pre-mRNA in mammalian and yeast extracts. Proc. Natl. Acad. Sci. 83: 2022-2026.

Rymond, B.C. and M. Rosbash. 1985. Cleavage of $5^{\prime}$ splice site and lariat formation are independent of $3^{\prime}$ splice site in yeast mRNA splicing. Nature 317: 735-737.

- 1986. Differential nuclease sensitivity identifies tight contacts between yeast pre-mRNA and spliceosomes. $E M B O$ J. 5: 3517-3523. 
Rymond, B.C., D.D. Torrey, and M. Rosbash. 1987. A novel role for the 3' region of introns in pre-mRNA splicing of Saccharomyces cerevisiae. Genes Dev. 1: 238-246.

Siebenlist, U. and W. Gilbert. 1980. Contact between Escherichia coli RNA polymerase and an early promoter of phage T7. Proc. Nat1. Acad. Sci. 77: 122-126.

Siliciano, P.G., P.A. Brow, H. Roiha, and C. Guthrie. 1987a. An essential snRNA from $S$. cerevisiae has properties predicted for U4, including interaction with a U6-like snRNA. Cell 50: $585-592$.

Siliciano, P.G., M.H. Jones, and C. Guthrie. 1987b. Saccharomyces cerevisiae has a U1-like small nuclear RNA with unexpected properties. Science 237: 1484-1487.

Vijayraghavan, U., R. Parker, J. Tamm, Y. Iimura, J. Rossi, J. Abelson, and C. Guthrie. 1986. Mutations in conserved intron sequences affect multiple steps in the yeast splicing pathway, particularly assembly of the spliceosome. EMBO J. 5: $1683-1695$

Zhuang, Y. and A.M. Weiner. 1986. A compensatory base change in Ul snRNA suppresses a $5^{\prime}$ splice site mutation. Cell 46: 827-835.

Zillmann, M., M.L. Zapp, and S.M. Berget. 1988. Gel-electrophoretic isolation of splicing complexes containing U1 snRNPs. Mol. Cell. Biol. 8: 814-821. 


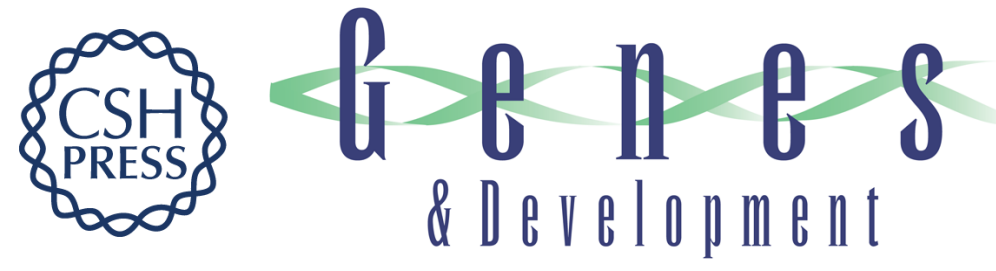

\section{A chemical modification/interference study of yeast pre-mRNA spliceosome assembly and splicing.}

B C Rymond and M Rosbash

Genes Dev. 1988, 2:

Access the most recent version at doi:10.1101/gad.2.4.428

References This article cites 42 articles, 15 of which can be accessed free at:

http://genesdev.cshlp.org/content/2/4/428.full.html\#ref-list-1

License

Email Alerting

Service

Receive free email alerts when new articles cite this article - sign up in the box at the top right corner of the article or click here.

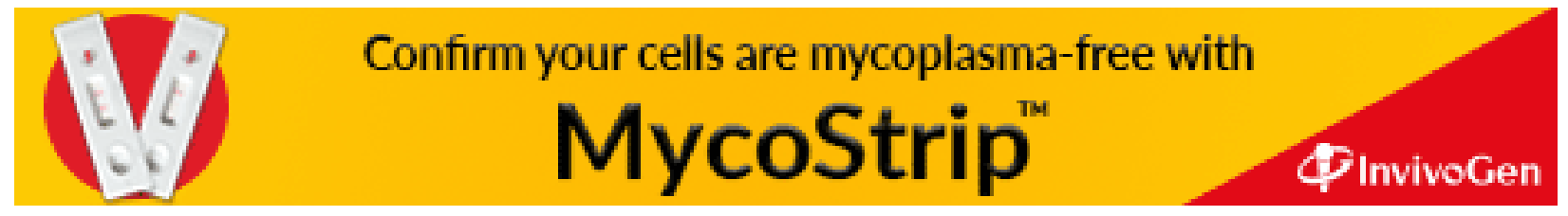

